\title{
Moving Forward: Gradual Return of Gastroenterology Practice during the COVID-19 Pandemic
}

\author{
Carlos Paolo Francisco lan Homer Cua Enrik John Aguila \\ Patricia Anne Cabral-Prodigalidad Marianne Linley Sy-Janairo \\ Joseph Erwin Dumagpi Nikko Theodore Raymundo Juliet Gopez-Cervantes \\ Jonard Co
}

Institute of Digestive and Liver Diseases, St. Luke's Medical Center Global City, Taguig City, Philippines

\author{
Keywords \\ COVID-19. Gl practice · Gastroenterology · Hepatology · \\ Endoscopy
}

\begin{abstract}
Background: The COVID-19 pandemic has caused disruption of routine gastroenterology practice, which has resulted in the suspension of elective endoscopic procedures and outpatient consults. For the past months, the strategy was to mitigate infection risk for the healthcare team while still providing essential service to patients. Prolonged suspension of the outpatient clinics and endoscopy practice, however, is deemed unsustainable and could even be detrimental. It can compromise patient care and result in poor outcomes; hence, a well-crafted plan is needed for the gradual resumption of clinic operations and endoscopic procedures. Summary: As the world begins to transition to the "new normal," there are new health and safety issues to consider. Adaptive measures like telemedicine and electronic health records should be utilized to facilitate patient care while minimizing exposure. Careful patient screening, adequate
\end{abstract}

karger@karger.com

(c) 2020 S. Karger AG, Basel

www.karger.com/ddi

Karger" supply of personal protective equipment, effective infection-control policies, as well as appropriate administrative modifications are needed for a safe return of gastroenterology practice. Key Messages: Ensuring the safety of patients, caregivers, and healthcare workers should remain as top priority. To help ease the transition as we move forward from this pandemic, we present a review of recommendations to guide gastroenterologists and endoscopy unit administrators in the gradual return to gastroenterology practice.

(c) 2020 S. Karger AG, Basel

\section{Introduction}

The COVID-19 pandemic has led to extensive economic damage resulting in millions of lost jobs and revenue reductions worldwide. The healthcare system has suffered an unprecedented challenge to stay afloat, given the huge impact felt on hospital systems, outpatient clinics, and ancillary units. A Harvard research showed a $60 \%$ decline in the number of visits to ambulatory practices since early April [1]. Hospital revenues have de- 
clined sharply as a result of the pandemic. Healthcare workers (HCWs) have raised concerns that patients are forgoing important care, such as chronic disease management, which can further jeopardize their health conditions [2]. For the practice of gastroenterology in particular, the effects of the pandemic have also led to delays in patient care with face-to-face consultations and elective endoscopy procedures having been postponed for several months already. Prolonged suspension of gastrointestinal (GI) services have resulted in many GI conditions, once allowed to wait, to now require more urgent attention.

As the crisis is foreseen to stay for a long time, this article provides different evidence-based strategies on the efficient and safe return to GI practice during the pandemic. This article can also help in the development of policies and recommendations that can guide gastroenterologists, hepatologists, and other healthcare professionals in moving forward towards the new normal.

\section{Methods}

This review is based on available literature relating to the strategies of effective and safe resumption of GI practice and endoscopic procedures published in different international gastroenterology and digestive endoscopy society Web sites: the American College of Gastroenterology (ACG), American Gastroenterological Association (AGA), American Society for Gastrointestinal Endoscopy (ASGE), American Association for the Study of the Liver (AASLD), British Society of Gastroenterology (BSG), Chinese Society of Gastroenterology (CSG), European Association for the Study of the Liver (EASL), European Society for Gastrointestinal Endoscopy (ESGE), Asian Pacific Society for Digestive Endoscopy (APSDE), and the Philippine Society of Digestive Endoscopy (PSDE). Relevant literatures were also reviewed from different public health Web sites such as the World Health Organization (WHO), US Centers for Disease Control (CDC), World Medical Association (WMA), American Medical Association (AMA), European Society of Clinical Microbiology and Infectious Diseases (ESCMID), Infectious Diseases Society of America (IDSA), Department of Health - Republic of the Philippines (DOH), Philippine Society for Microbiology and Infectious Diseases (PSMID), and other government health agency Web sites. Most of these recommendations are based on expert consensus, as rigorous data are not yet available. Extensive hand-searching of the reference lists of manuscripts and cross-referencing were done.

\section{Results}

After extensive literature search, 5 domains of interest were identified to be of greatest significance regarding the resumption of GI practice and endoscopic procedures.
These areas of concern focus on the difficulty of face-toface consultations, managing inpatients, conducting outpatient clinics, reopening endoscopy units, and caring for patients with special issues such as liver disease.

\section{Limiting Physical Encounters and the Role of}

\section{Telemedicine in the GI Practice}

To limit physical encounter, the practice of telemedicine has served as a viable alternative, and its use has grown fast since the onset of the COVID-19 pandemic. It is defined as the practice of medicine over a distance, in which interventions, diagnostic and treatment decisions, and recommendations are based on data, documents, and other information transmitted through telecommunication systems [3]. Its practice is supported by different government legislations and medical societies worldwide. Hospitals, doctors' associations, and societies have launched online platforms, which allow patients to consult initially with doctors before seeking face-to-face consultation [4]. In the context of the pandemic, it has replaced the usual face-to-face physician-patient consults as its benefits outweigh the risks especially for vulnerable patients (e.g., comorbid or immunocompromised patients) [5]. Given its noncontact nature, this strategy has proven to be effective in assuring physical distancing and mitigating unnecessary exposures [6].

Telemedicine offers a particular advantage for the follow-up of patients with inflammatory bowel disease and chronic liver conditions such as hepatocellular carcinoma (HCC) and post-liver transplantation [7]. Patients with nonurgent GI complaints can also be assisted through instructions and be given medications during their virtual consults. The Philippine Food and Drug Administration provided guidelines for issuance of e-prescriptions [4]. While telemedicine is seen to be primarily used for outpatient consultations, another of its important functions is the triaging for endoscopic procedures. According to the ASGE, it should be considered for each patient referred for endoscopy to be able to assess comorbidities and possible exposure to COVID-19 [8]. During the consult, the procedure and pre-procedural instructions such as the bowel preparation can be explained. Expectations on the day of the procedure can also be discussed. In such way, the patient's actual stay in the endoscopy unit would be shorter.

The practice of telemedicine is governed by the principles of accountability, confidentiality, and ethics. These should be observed by the physician at all phases. In setting up a telemedicine service, special attention should be given to ensuring transparency of information to pa- 
tients, obtaining true informed consent, guaranteeing storage of sensitive personal information, observing strict security requirements, and protocol breach. The telemedicine platform should be secure and accessible. Informed consent should ensure that the patient understands the benefits and risks of treatment and the medium through which care will be provided.

It is important to note that telemedicine may not be the appropriate model of care for every patient as resources and access to requisite technology is required [9]. Furthermore, it is inappropriate for encounters where physical examination is crucial, especially in situations where the risks of the coronavirus is absent. Although imperfect, drastic situations have called for its use, and it will always be a good alternative during the pandemic.

\section{Reopening of Outpatient Clinics}

Clinic Setup

Appropriate precautions are necessary to take in creating a safe and protected area for physicians, staff, and patients. These include assessing exposure risk, consideration of transmission routes, and establishing appropriate controls.

Proper and rational use of personal protective equipment (PPE), hand hygiene, and physical distancing are the primary strategies to prevent exposure [10]. Physical barriers to reduce exposure to the COVID-19 virus, such as glass or acrylic or windows are recommended. No studies, however, have been done regarding their use in the outpatient clinic setting. If barriers will be used, it is recommended that they are regularly cleaned and disinfected $[5,11,12]$. Maintaining adequate ventilation is another important precaution. At least 6-12 air changes per hour are recommended, which can be achieved through natural or mechanical ventilation. A high-efficiency particulate air filter may be used to improve air quality if adequate ventilation cannot be achieved $[5,12]$.

Maintaining physical distance of at least 6 feet remains to be an important strategy to minimize exposure and transmission. A recent meta-analysis showed that physical distancing of at least $1 \mathrm{~m}$ is strongly associated with protection, but $2 \mathrm{~m}$ might be more effective [13]. Healthcare units are advised to limit the number of people inside their facility. A one-way traffic flow for patients and staff is a helpful strategy. Installing visual markers can guide patient flow and enforce social distancing $[5,12,14]$.

\section{Clinic Staff}

Prior to returning to work, all clinic staff are advised to be screened for symptoms of COVID-19. A standard screening form for symptoms, travel, and contact history may be used for this purpose. Symptomatic staff with a history of travel or exposure must not be allowed to return to work and must seek immediate consult. There is currently no consensus or guideline on routine testing for COVID-19 of asymptomatic staff prior to returning to work. Daily temperature and symptom monitoring are recommended by the CDC. All clinic staff members are advised to have an updated immunization record $[5,11$, $14,15]$. It is important that they are knowledgeable of the healthcare facility's protocol should someone present with symptoms of COVID- $19[5,11,14]$. To reduce transmission, the recommended PPE for physicians and clinic staff are the following: face mask and goggles or face shield. N95 masks are only recommended if aerosol-generating procedures will be done. Coveralls or hazmat suits, hair caps, and shoe covers are not recommended [5, 16].

\section{Scheduling and Appointments}

Face-to-face clinic consultations are recommended to be done only by appointment. Patients are advised to seek initial consultation by telemedicine; however, in cases of GI bleeding or jaundice, inpatient consultation is recommended. If face-to-face consultation is necessary, screening for symptoms will be done online or via phone $[5,15]$. A health declaration form must be submitted by the patient and their companion before entering the hospital premises as shown in Figure 1. At the healthcare facility, a "no-mask, no-entry policy" is recommended in conjunction with temperature check at the point of entry. Viral testing for SARS-CoV-2 is recommended among individuals with symptoms consistent with COVID-19 or asymptomatic individuals with known or suspected exposure [17]. CDC recommends implementing universal source control measures for everyone in a healthcare facility because of the potential for asymptomatic and presymptomatic transmission. All patients should wear cloth face coverings or facemasks to prevent the spread of respiratory secretions when they are talking, sneezing, or coughing [18]. Only one companion is allowed if necessary (e.g., minors, elderly, and handicapped) $[5,14]$. Patient encounter should be kept at a minimum to limit exposure. Adequate interval between patients is advised to allow disinfection [5]. The use of electronic health records, prescriptions, diagnostic request forms, and other documents are recommended. Electronic or online payment mechanisms are also encouraged. 
Fig. 1. Health declaration form.

\begin{tabular}{|l|l|l|l|}
\hline Please tick an answer for every question item & YES & NO \\
\hline Have you been recently tested for COVID-19? & & \\
\hline Date swabbed: & & \\
\hline Have you been evaluated as Probable or Suspected for COVID-19? & & \\
\hline If YES, when did your quarantine start? & & \\
\hline $\begin{array}{l}\text { Do you have any travel history in the past 14 days? } \\
\text { If YES, when and where? }\end{array}$ & & \\
\hline $\begin{array}{l}\text { Did you come in close contact or staying in the same close environment } \\
\text { with someone who is a confirmed COVID-19 case? }\end{array}$ & & \\
\hline Did you come in close contact with a Probable or Suspected person with COVID-19? & & \\
\hline $\begin{array}{l}\text { Have you experienced the following symptoms recently? } \\
\text { Fever (>38 }{ }^{\circ} \text { C) }\end{array}$ & & \\
\hline Diarrhea, Nausea, or Vormiting & & \\
\hline Shortness of breath or other respiratory symptoms & & \\
\hline Other respiratory symptoms & & \\
\hline Headache & & \\
\hline Joint Pain or Muscle Pain & & \\
\hline Flu-like symptoms such as: \\
Chills or repeated shaking with chills & & \\
\hline Body aches & & \\
\hline Sore throat & & \\
\hline Runny Nose or Sneezing & & \\
\hline Cough and colds & & \\
\hline New loss of smell and/or taste & & \\
\hline Eye discharge & & \\
\hline Skin rash or discoloration of toes/fingers & & \\
\hline Loss of speech or movement & & \\
\hline
\end{tabular}

Cleaning and Disinfection

SARS-CoV-2 can remain viable for $3 \mathrm{~h}$ in aerosols and up to days on plastic surfaces; therefore, fomite transmission is possible [19]. Hand hygiene remains to be the cornerstone in infection control. Handwashing using soap and water is strongly recommended, otherwise hand hygiene with alcohol-based hand rubs with at least $70 \%$ alcohol may be done $[5,12,14]$. Hospital-grade cleaners are recommended for use in the clinic's equipment and surfaces, followed by disinfection that eliminates most pathogenic microorganisms. High-touch surfaces and objects like doorknobs, tabletops, light switches, computer keyboards, stethoscopes, and pens should be disinfected after every clinic session. Use of ultraviolet germicidal irradiation is an adjunct to standard disinfection procedures. There is currently no evidence to recommend the use of foot/shoe baths or disinfection mats in the outpatient clinic $[5,12]$.

\section{Role of Group Practice in Managing Inpatients}

Due to the risk of prolonged exposure and the limited supply of PPE, physicians have had to adjust their prac- tices without sacrificing the quality of patient care. Most physicians coming from private institutions have shifted into group practice. Group practice is defined by the AMA as " 3 or more physicians formally organized as a legal entity in which business, clinical, and administrative facilities are shared" [20]. This shift in the structure of health care has grown and changed the composition and management in patient care [21].

Benefits in the setting of single-specialty group practice include profitability, improved lifestyle, and enhanced quality of patient care [20]. Having more physicians lessen the burden of the business aspect in medical practice [22]. Resources can be pooled, and with consolidation, the group can provide profitable procedures and other ancillary services [21]. Data from a survey in Taiwan showed that patients perceive better service quality with group practices compared with solo practices on all dimensions [23]. Group practice also creates an environment of organizational accountability regarding skills, clinical judgment, and patient care [20]. 
Table 1. Recommended precautions and PPE based on local prevalence, COVID-19 RT-PCR test, and symptom screen

\begin{tabular}{llll}
\hline Prevalence & COVID-19 RT-PCR test & Symptom screen & Precaution \\
\hline Low & Negative & Negative & Standard precautions $^{\mathrm{a}}$ \\
Low & Not available & Negative & Enhanced precautions $^{\mathrm{b}}$ \\
Low & Positive & Negative or positive & Delay procedure or maximum precautions ${ }^{\mathrm{c}}$ \\
High & Negative & Negative & Enhanced precautions $^{\mathrm{b}}$ \\
High & Not available & Negative & Maximum precautions \\
High or low & Positive & Positive & Delay procedure or maximum precautions ${ }^{\mathrm{c}}$ \\
\hline
\end{tabular}

PPE, personal protective equipment; RT-PCR, reverse transcription polymerase chain reaction. ${ }^{\text {a }}$ Standard precautions: full-sleeved gown, face shield/goggles, gloves, and surgical mask. ${ }^{\mathrm{b}}$ Enhanced precautions: hairnet, shoe covers, full-sleeved gown, face shield/goggles, gloves, and N95 respirator. ${ }^{\mathrm{c}}$ Maximum precautions: negative pressure room, hairnet, shoe covers, full-sleeved gown, face shield/goggles, gloves, and N95 respirator.

Autonomy, decision-making abilities, differences in work styles, and clinical decisions may be present and cannot be avoided, and this may bring conflict within the group. These disadvantages, however, can be made irrelevant with effective communication and proper coordination to settle certain decisions within the group [24]. In the practice of endoscopy, especially for semiurgent and elective cases, pre-procedure team discussion greatly assists in decision-making regarding the necessity and prioritization of procedures, treatment planning, and goals. Post hoc discussion of cases also benefit the team with regard to follow-up care. Applying this in practice makes better use of each individual's knowledge and skills.

\section{Gradual Reopening of Endoscopy Units}

Since the declaration of COVID-19 as a pandemic, restrictive measures have been implemented worldwide to prevent the spread of the virus. Different GI and digestive societies have recommended to limit endoscopy to emergency and time-sensitive procedures in order to minimize risk of infection among staff and preserve PPE. Endoscopic procedures have been suspended due to its assumed aerosol-generating properties putting HCWs in endoscopy units at a higher risk [25]. This has actually been proven in a recent proof-of-concept study that revealed doing an esophagogastroduodenoscopy indeed increases the amount of generated aerosols [26]. Colonoscopy on the contrary is considered a low-aerosolgenerating procedure. With these propositions, conduct of endoscopic procedures has been largely limited since the beginning of the pandemic. Unfortunately, these mitigation efforts have left unintended healthcare consequences such as delays in diagnosis and management.
According to a recent report, suspension of elective colonoscopy for 6 months may result in the delayed diagnosis of over 2,800 colorectal cancers and 22,000 high-grade adenomatous polyps in the USA alone [27, 28]. Needless to say, further suspension of elective digestive endoscopy may translate to dire outcomes for other GI pathologies as well.

According to the APSDE, stepwise resumption of elective endoscopy services should be guided by control of COVID-19 in the local community, availability of manpower, and equipment supply. A modified endoscopy unit operation policy and workflow should be established to prevent hospital-based transmission and cross contamination $[29,30]$. Assigning patients to a predefined priority stratification groups will differentiate between procedures that should be performed immediately and rescheduled. Forming an ad hoc committee is recommended for case-by-case evaluation of endoscopic procedures based on medical priority [27, 31-33]. Elective endoscopy may be considered when there is a downtrend in new cases of COVID-19 and an adequate reserve of PPE for 8 weeks. Endoscopy units may resume full capacity when there is no new case of COVID-19 for at least 2 weeks [29]. It is important that a special consent form regarding COVID-19 and the potential risk of transmission related to entering a medical facility and undergoing a medical procedure should be signed [31]. After endoscopy, phone follow-up at 7 and 14 days post-procedure is recommended to monitor any development of COVID-19 symptoms [32-34]. Standard disinfection algorithms and reprocessing protocols of endoscopes are sufficient since most enzymatic solutions can eliminate SARS-CoV-2 [23, 34, 35]. 
Safe Endoscopy and Practical Use of PPEs

A recent international consensus study for the resumption of endoscopy services has defined high-prevalence regions as areas with estimated disease prevalence rates of $2 \%$ or higher [36]. For these regions, stricter guidelines are required in the practice of endoscopy and use of PPE. In general, PPE requirements depend on the local prevalence of COVID-19, availability of a COVID-19 screening test, and patient symptoms as shown in Table 1. Current guidelines recommend the use of full PPE for all emergency and urgent procedures, this includes N95/FFP2/FFP3 respirator, hairnet, goggles/face shield, full-sleeve isolation gown, gloves, and shoe covers. In areas with low prevalence, the use of standard PPE is sufficient for low-aerosol-generating procedures such as colonoscopy [36]. A standard PPE encompasses fullsleeved gown, protective eye wear (face shield/goggles), gloves, and a surgical mask. A retrospective study in Italy showed a lower infection rate (4.3\%) among HCWs in an endoscopy center with adequate PPE than the average infection rate (10\%) among HCWs [37].

Recommendations vary only slightly regarding preprocedure testing and PPE requirements for nonurgent cases. Most authors will suggest that in the transition to the new normal and the reopening of endoscopy units in low-prevalence areas, "standard" precautions may be observed in performing elective endoscopy on patients with negative symptom screen and negative reverse transcription-PCR (RT-PCR) COVID-19 testing. While most consider pre-procedure testing as ideal, many acknowledge the absence of widespread reliable and accurate rapid testing, and because of this, the use of N95 masks ("enhanced" and "maximum" precautions) while performing GI endoscopic procedures regardless of patient's community disease prevalence has been offered as an acceptable option $[27,28]$.

Elective endoscopy in high-prevalence areas should be performed under enhanced precautions with N95 masks at a minimum $[27,28]$. One study from China showed that no medical staff who wore N95 respirators and practiced strict hand hygiene were infected despite being exposed in high-risk departments [38]. For nonurgent cases, regardless of prevalence, those with positive COVID-19 test or symptom screen should have their procedure delayed [27, 28, 33, 34, 39-43].

A study conducted in South Korea during the Ebola outbreak reported that significant number of contaminations occur during doffing, and the most vulnerable process was removing respirators (79.2\%) [44]. Endoscopy staff must receive proper education and training regard-

Gradual Return of Gastroenterology

Practice during the COVID-19 Pandemic ing the use of PPE to minimize protocol breach since selfcontamination is frequently encountered $[11,45]$. A buddy system would be of help in preventing protocol breach by confirming that PPE is properly doffed [25].

\section{Asymptomatic Transmission and RT-PCR}

COVID-19 Testing

The absence of clinical symptoms does not exclude SARS-CoV-2 infection. Evidence has shown possible disease transmission even from asymptomatic individuals; hence, screening patients based on symptoms, travel history, or exposure may be insufficient [36]. The IDSA panel recommends testing asymptomatic individuals scheduled for a time-sensitive procedure as close to the planned procedure as possible [43]. Other guidelines recommend that testing be performed within $24-72 \mathrm{~h}$ prior to an endoscopic procedure, with patient self-isolating from the time of testing to time of procedure $[27,36]$. In a recent study from China, no endoscopy-related COVID-19 infection transmission has been reported after following strict screening protocols [46].

In general, testing patients prior to elective endoscopy should be individualized based on community disease prevalence, local availability of testing, adequate turnaround time, and sensitivity. While it could be reasoned that asymptomatic patients from low-prevalence areas may not require a costly COVID-19 RT-PCR testing, a recently published study found a positive economic impact in testing all patients for COVID-19 [47]. Routine COVID-19 RT-PCR testing for staff and physicians is currently not recommended.

\section{Care of Patients with Liver Disease}

Everyone is considered to be susceptible to SARSCoV-2 infection, but older patients and those with preexisting medical conditions are considered to be at higher risk. The extent of the risk of patients with liver disease remains uncertain [48]. Several studies showed that patients with metabolic associated fatty liver disease are associated with higher risk for a severe illness [49-51]. Similarly, patients with cirrhosis, HCC, autoimmune liver disease, and liver transplant recipients are potentially at greater risk for severe COVID-19 [52]. Among COVID-19 patients, 23 and $2 \%$ were observed to have elevated alanine aminotransferase (ALT)/aspartate aminotransferase (AST) and acute liver injury, respectively. Hepatotoxicity by commonly used off-label COVID-19 medications can contribute to liver injury. These situations can even be complicated by preexisting chronic liver diseases. Regular monitoring of liver biochemistries is 
vital since liver-related complications, overall complications, and outcomes correlate with the existing hepatic reserve $[53,54]$.

Outpatient Care for Liver Disease Patients

Care of patients with chronic liver conditions may be done via telemedicine including recipients of liver transplant [55]. Hospital visits should be postponed while COVID-19 is prevalent in the community. Face-to-face consultations may be done only when deemed necessary to be assessed. Routine laboratory testing or other workups may also be done in an outpatient setting to limit exposure inside the hospital. The AASLD recommends seeing only new patients with urgent issues and clinically significant liver disease such as jaundice, significantly elevated liver enzymes (ALT or AST > $500 \mathrm{U} / \mathrm{L}$ ), recent-onset hepatic decompensation, and selected patients with liver cancer or for liver transplant evaluation [47].

Procedures for Liver Disease Patients

Noninvasive risk assessment for the presence of varices may be utilized in order to stratify patients who need to undergo endoscopic screening. Among patients with compensated cirrhosis, surveillance for HCC may be delayed for up to 3 months since annual screening also provides benefits with no difference in overall survival [55]. Patients with increased risk, those with elevated alphafetoprotein, advanced cirrhosis, and chronic hepatitis B infection may be prioritized [52]. Elective endoscopic variceal screening is recommended to be postponed in patients with no history of GI bleeding until the COVID-19 outbreak is under control [55]. The EASL and AASLD guidelines recommend performing screening endoscopy only for patients at high risk for bleeding such as those with a history of variceal bleeding or signs of portal hypertension (ascites and thrombocytopenia) [48, 52]. Patients with HCC should continue treatment and radiologic surveillance as scheduled. In elderly patients and those at higher risk for severe COVID-19 illness, however, screening can be postponed for up to 3 months [56].

According to EASL, indications for liver biopsy during this pandemic include significantly elevated transaminases (e.g., ALT > 5× ULN) of unknown etiology and liver masses suspicious of malignancy. It is advised to defer liver biopsy for the diagnosis of metabolic associated fatty liver disease or for grading or staging of chronic viral hepatitis [48].

Living-donor transplantations should be considered on a case-by-case basis [52]. EASL and AASLD recommend the following as listing for transplantation: patients with poor short-term prognosis including those with acute/acute-on-chronic liver failure, high model for endstage liver disease score, and HCC at the upper limits of the Milan criteria. COVID-19-positive candidates are not recommended to proceed with liver transplant. They may be considered for transplantation at least 14-21 days after symptom resolution and 1 or 2 negative SARS-CoV- 2 diagnostic tests [48, 52].

AASLD recommends to proceed with liver cancer treatments and if possible surgical resection in patients with HCC [52]. Palliative treatments, such as transarterial chemoembolization or radioembolization are continued, but is recommended to be postponed in the elderly, weighing the oncological benefit versus the risk of exposure to SARS-CoV-2 [56].

\section{Discussion}

The COVID-19 pandemic has made a dramatic impact in the practice of gastroenterology. The rapid spread of the virus has led to temporary suspension of nonessential GI functions and elective endoscopic procedures worldwide. As current mitigation efforts take effect, it is imperative that GI institutions establish clear plans and standard operating procedures in adapting to the new normal. After having identified the areas of greatest significance regarding the resumption of GI practice, we summarize the following practical recommendations for each domain of interest as shown in Table 2. The societies supporting such strategy are also indicated.

Telemedicine may not be the appropriate model of care for every patient as the need for resources and access to requisite technology is required [9]. However, it is a valued alternative to the usual face-to-face consults for nonurgent GI complaints, as its benefits outweigh risks, especially for older patients and those with preexisting medical conditions [5]. It should also be considered in screening patients referred for endoscopic procedures.

Outpatient clinic consultations may be done after careful planning [15]. All patients and staff are recommended to be screened for potential symptoms. Although routine testing of asymptomatic staff prior to their return to work has not been recommended, patients and staff must continue to observe strict physical distancing and hand hygiene [27]. Sufficient facilities and resources such as PPE must be available for the protection of all.

Setting up a group practice during this seemingly difficult time of the pandemic is advantageous not only for patients but also for physicians. When a harmonious re- 
Table 2. Summary of key strategies and recommendations in the resumption of GI practice

\begin{tabular}{|c|c|c|}
\hline Domain of interest & Society & Key strategies and recommendations \\
\hline $\begin{array}{l}\text { Telemedicine in } \\
\text { the GI practice }\end{array}$ & $\begin{array}{l}\text { ASGE, WMA, and } \\
\text { PSMID }\end{array}$ & $\begin{array}{l}\text { - Consider telemedicine in nonurgent cases and for triaging patients referred for endoscopic procedures } \\
\text { - Ensure that patients understand the benefits and risks of treatment and the medium through which care } \\
\text { will be provided }\end{array}$ \\
\hline $\begin{array}{l}\text { Reopening of } \\
\text { outpatient clinics }\end{array}$ & $\begin{array}{l}\text { AMA, CDC, DOH, } \\
\text { WHO, and PSMID }\end{array}$ & $\begin{array}{l}\text { - Observe proper and rationale use of PPE, hand hygiene, and physical distancing to minimize risk and } \\
\text { exposure } \\
\text { - Educate staff on the signs and symptoms of COVID-19, infection control policies, and protocols } \\
\text { - Adopt electronic health records, prescriptions, laboratory request forms, and noncontact payment } \\
\text { mechanisms }\end{array}$ \\
\hline $\begin{array}{l}\text { Group practice in } \\
\text { the management } \\
\text { of inpatients }\end{array}$ & AMA & $\begin{array}{l}\text { - Pursue group practice to preserve manpower and resources, especially invaluable supplies of PPE } \\
\text { - Establish effective communication and proper coordination as these are key to settle and address issues } \\
\text { within the group }\end{array}$ \\
\hline $\begin{array}{l}\text { Reopening of } \\
\text { endoscopy units }\end{array}$ & $\begin{array}{l}\text { ACG, AGA, APSDE, } \\
\text { ASGE, BSG, CSG, } \\
\text { ESGE, IDSA, and } \\
\text { PSDE }\end{array}$ & $\begin{array}{l}\text { - Consider local community disease control, staff availability, and adequate resources in the gradual } \\
\text { resumption of elective endoscopy } \\
\text { - Modify endoscopy unit operation policies and workflow to observe recommendations on safety, rational } \\
\text { use of PPE, and testing } \\
\text { - Perform pre-procedure screening prior to scheduling of procedures }\end{array}$ \\
\hline $\begin{array}{l}\text { Care of patients } \\
\text { with liver disease }\end{array}$ & AASLD, EASL & $\begin{array}{l}\text { - Use noninvasive risk assessment tools to stratify patients regarding the need to undergo endoscopic } \\
\text { screening for varices } \\
\text { - In patients with compensated cirrhosis, surveillance for HCC and screening for varices may be delayed for } \\
\text { up to } 2 \text { months } \\
\text { - For HCC, continue treatment and radiologic surveillance as scheduled. Postpone for elderly and those at } \\
\text { higher risk for severe COVID-19 illness }\end{array}$ \\
\hline
\end{tabular}

ASGE, American Society for Gastrointestinal Endoscopy; WMA, World Medical Association; PSMID, Philippine Society for Microbiology and Infectious Diseases; AMA, American Medical Association; CDC, US Centers for Disease Control; WHO, World Health Organization; ACG, American College of Gastroenterology; APSDE, Asian Pacific Society for Digestive Endoscopy; AGA, American Gastroenterological Association; BSG, British Society of Gastroenterology; CSG, Chinese Society of Gastroenterology; DOH, Department of Health - Republic of the Philippines; ESGE, European Society for Gastrointestinal Endoscopy; IDSA, Infectious Diseases Society of America; PSDE, Philippine Society of Digestive Endoscopy; AASLD, American Association for the Study of the Liver; EASL, European Association for the Study of the Liver; GI, gastrointestinal; HCC, hepatocellular carcinoma; PPE, personal protective equipment.

lationship between members is maintained, it creates an environment for organized patient care [20]. Team discussions employing effective communication and proper coordination can settle certain patient issues and treatment dilemmas not otherwise easily reached by an individual physician alone [24].

Stepwise resumption of elective endoscopy should be guided by control of COVID-19 in the local community, availability of manpower, and equipment supply [29]. Every effort to minimize hospital-based transmission via the establishment of carefully modified unit and infection control policies must be undertaken [30,31].

Management of special patient populations, including patients with preexisting liver conditions, needs careful consideration. The urgency for surveillance and screening must be weighed against existing knowledge regarding the risks of COVID-19.

As thoroughly discussed, several factors should be considered in the gradual return of gastroenterology practice during the pandemic. With the guidelines changing frequently due to the protean features of COVID-19, we acknowledge the limitation of being only able to consolidate current recommendations of major international gastroenterology, digestive endoscopy, and public health societies from different regions of the world. Nevertheless, we recognize that it is also important to observe the local guidelines whenever available.

In conclusion, as the COVD-19 pandemic is slowly coming under control, gastroenterologists need to answer the questions of when and how to safely resume practice. The gradual resumption of clinical and endoscopic operations must follow a well-thought-of and stepwise protocol. Plans should be well-crafted to carefully navigate possible obstacles. Recommendations are available and will help ease the transition to the new normal but it is important to recognize, however, that these may not be applicable in all settings and may change as the situation evolves. The decision to reopen facilities should 
be based on local disease prevalence, workforce capacity, adequate supplies, and the consideration of local health agency and institutional recommendations.

\section{Conflict of Interest Statement}

The authors have no conflicts of interest to declare.

\section{Funding Sources}

The authors did not receive any funding.

\section{Author Contributions}

All authors have contributed to and agreed on the content of the manuscript. C.P. Francisco, I.H. Cua, E.J. Aguila, M.L. Sy-Janairo, J.E. Dumagpi, N.T. Raymundo, and P.A. Cabral-Prodigalidad contributed in literature search, study design, data collection, data analysis, data interpretation, and writing of the manuscript. I.H. Cua, C.P. Francisco, and E.J. Aguila conceived the study. C.P. Francisco created the figure. P.A. Cabral-Prodigalidad, I.H. Cua, C.P. Francisco, E.J. Aguila, J. Co, and J. Gopez-Cervantes critically reviewed the content of the paper. All authors discussed and contributed to the final manuscript. C.P. Francisco, E.J. Aguila, and P.A. Cabral-Prodigalidad finalized the manuscript.

\section{References}

1 Mehrota A, Chernew M, Linetsky D, Hatch H, Cutler D. The impact of the COVID-19 pandemic on outpatient visits: a rebound emerges [Internet]. 2020.

2 Hospitals and health systems face unprecedented financial pressures due to COVID-19. Am Hosp Assoc. 2020 [cited 2020 Jul 29]. Available from: https://www.aha.org/ guidesreports/2020--05--05-hospitals-andhealth-systems-face-unprecedented-financial-pressures-due.

3 WMA: the world medical association. WMA statement on the ethics of telemedicine [Internet]. https://www.wma.net/policies-post/ wma-statement-on-the-ethics-of-telemedicine/.

4 Acosta F, Siao K, Resurreccion E. Addressing COVID-19 with healthcare in a digital economy [Internet]. 2020.

5 Roxas E, Roman A, Garcia J, Zabat G, Caoili J, Gregorio K, et al. Infection prevention and control guidelines for outpatient clinic resumption in the context of COVID-19 [Internet]. 2020.

6 Keesara S, Jonas A, Schulman K. Covid-19 and health care's digital revolution. $\mathrm{N}$ Engl J Med. 2020;382(23):e82.

7 Fix OK, Hameed B, Fontana RJ, Kwok RM, McGuire BM, Mulligan DC, et al. Clinical best practice advice for hepatology and liver transplant providers during the COVID-19 pandemic: AASLD expert panel consensus statement. Hepatology. 2020; 72(1):287-304. https://doi.org/10.1002/hep.31281.

8 Hennessy B, Vicari J, Bernstein B, Chapman F, Khaykis I, Littenberg G, et al. Guidance for resuming GI endoscopy and practice operations after the COVID-19 pandemic. Gastrointest Endosc. 2020;92(3):743-7.e1.

9 Chaet D, Clearfield R, Sabin JE, Skimming K. Ethical practice in telehealth and telemedicine. J Gen Intern Med. 2017;32(10):1136-40.

10 Re-opening facilities to provide non-emergent non-COVID-19 healthcare: phase I [internet]. Cms.gov. 2020 [cited 2020 May 18]. Available from: https://www.cms.gov/files/ document/covid-flexibility-reopen-essentialnon-covid-services.pdf.
11 Coronavirus disease 2019 (COVID-19) [Internet]. Centers for Disease Control and Prevention. 2020 [cited 2020 May 18]. Available from: https://www.cdc.gov/coronavirus/ 2019-ncov/hcp/clinic-preparedness.html.

12 Severe acute respiratory infections treatment centre [Internet]. Who.int. 2020 [cited 2020 May 18]. Available from: https://www.who. int/publications/i/item/severe-acute-respiratory-infections-treatment-centre.

13 Chu D, Akl E, Duda S, Solo K, Yaacoub S, Schünemann $\mathrm{H}$, et al. Physical distancing, face masks, and eye protection to prevent personto-person transmission of SARS-CoV-2 and COVID-19: a systematic review and metaanalysis. Lancet. 2020;395(10242):1973-87.

14 Interim guidelines on the return-to-work [internet]. Doh.gov.ph. 2020 [cited 2020 May 15]. Available from: https://www.doh.gov. $\mathrm{ph} / \mathrm{sites} / \mathrm{default} /$ files/health-update/ dm2020--0220.pdf.

15 COVID-19: a physician practice guide to reopening [Internet]. Am Medical Assoc. 2020 [cited 2020 May 21]. Available from: https:// www.ama-assn.org/delivering-care/publichealth/covid-19-physician-practice-guidereopening.

16 Rational use of personal protective equipment (PPE) for coronavirus disease (COVID-19): interim guidance, 19 May 2020 [Internet]. Apps.who.int. 2020 [cited 2020 May 21]. Available from: https://apps.who.int/iris/ handle/10665/331498.

17 Overview of testing for SARS-CoV-2 [Internet]. Centers for Disease Control and Prevention. 2020 [cited 2020 Aug 16]. Available from: https: //www.cdc.gov/coronavirus/ 2019-ncov/hcp/testing-overview.html.

18 Interim infection prevention and control recommendations for healthcare personnel during the coronavirus disease 2019 (COVID-19) pandemic [Internet]. Centers for Disease Control https://www.cdc.gov/coronavirus/ 2019-ncov/hcp/infection-control-recommendations.html.
19 van Doremalen N, Bushmaker T, Morris DH, Holbrook MG, Gamble A, Williamson BN, et al. Aerosol and surface stability of SARSCoV-2 as compared with SARS-CoV-1. N Engl J Med. 2020;382(16):1564-7.

20 Kash B, Tan D. Physician group practice trends: a comprehensive review. J Hosp Med Manage. 2016;2(1).

21 Liebhaber A, Grossman JM. Physicians moving to mid-sized, single-specialty practices. Track Rep. 2007(18):1-5.

22 Damiani G, Silvestrini G, Federico B, Cosentino M, Marvulli M, Tirabassi F, et al. A systematic review on the effectiveness of group versus single-handed practice. Health Policy. 2013;113(1-2):180-7.

23 Lin HC, Xirasagar S, Laditka JN. Patient perceptions of service quality in group versus solo practice clinics. Int J Qual Health Care. 2004;16(6):437-45

24 Types of medical practices: advantages \& disadvantages [Internet]. Study.com. 2020 [cited 2020 May 15]. Available from: https://study. com/academy/lesson/types-of-medical-practices-advantages-disadvantages.html.

25 Soetikno R, Teoh AYB, Kaltenbach T, Lau JYW, Asokkumar R, Cabral-Prodigalidad P, et al. Considerations in performing endoscopy during the COVID-19 pandemic. Gastrointest Endosc. 2020;92(1):176-83.

26 Chan S, Ma T, Ka-Chun Chong M, Chan D, Ng E, Chiu P. A proof of concept study: esophagogastroduodenoscopy is an aerosolgenerating procedure and continuous oral suction during the procedure reduces the amount of aerosol generated. Gastroenterology. 2020.

27 The American College of gastroenterology (ACG) roadmap for safely resuming or ramping-up endoscopy in the COVID-19 pandemic https://webfiles.gi.org/docs/policy/ 2020resuming-endoscopy-fin-05122020.pdf.

28 Gupta S, Shahidi N, Gilroy N, Rex D, Burgess N, Bourke M. Proposal for the return to routine endoscopy during the COVID-19 pandemic. Gastrointest Endosc. 2020;92(3):735742. 10.1016/j.gie.2020.04.050. 
29 Chiu PWY, Ng SC, Inoue H, Reddy DN, Ling Hu E, Cho JY, et al. Practice of endoscopy during COVID-19 pandemic: position statements of the Asian Pacific society for digestive endoscopy (APSDE-COVID statements). Gut. 2020;69(6):991-6.

30 Cennamo V, Bassi M, Landi S, Apolito P, Ghersi S, Dabizzi E, et al. Redesign of a GI endoscopy unit during the COVID-19 emergency: a practical model. Dig Liver Dis. 2020;52(10): 1178-87.

31 Guidance for resuming GI endoscopy and practice operations after the COVID-19 pandemic [Internet]. Asge.org. 2020 [cited 2020 May 15]. Available from: https://www.asge. org/docs/default-source/default-documentlibrary/asge-guidance-for-reopeningl_428-2020.pdf.

32 Repici A, Maselli R, Colombo M, Gabbiadini R, Spadaccini M, Anderloni A, et al. Coronavirus (COVID-19) outbreak: what the department of endoscopy should know. Gastrointest Endosc. 2020;92(1):192-7.

33 Gralnek IM, Hassan C, Beilenhoff U, Antonelli G, Ebigbo A, Pellisè M, et al. ESGE and ESGENA position statement on gastrointestinal endoscopy and the COVID-19 pandemic. Endoscopy. 2020;52(6):483-90.

34 PSDE clinical and procedural guidance for the resumption of semi-urgent and elective procedures at the http://www.psde.org.ph/ wp-content/uploads/2020/05/FINAL-PSDEGuidance-on-Resumption-of-Semi-electiveand-Elective-Procedures.pdf.

35 Kongkam P, Tiankanon K, Ratanalert S, Janthakun V, Kitiyakara T, Angsuwatcharakon $\mathrm{P}$, et al. The practice of endoscopy during the COVID-19 pandemic: recommendations from the Thai association for gastrointestinal endoscopy (TAGE) in collaboration with the endoscopy nurse society (Thailand). SMJ. 2020;72(4):283-6.

36 Bhandari P, Subramaniam S, Bourke M, Alkandari A, Chiu P, Brown J, et al. Recovery of endoscopy services in the era of COVID-19: recommendations from an international Delphi consensus. Gut. 2020;69(11):1915-24.

37 Repici A, Aragona G, Cengia G, Cantù P, Spadaccini M, Maselli R, et al. Low risk of covid-19 transmission in GI endoscopy. Gut. 2020; 69(11): 1925-27. http: //dx.doi.org/ 10.1136/gutjnl-2020-321341.
38 Wang X, Pan Z, Cheng Z. Association between 2019-nCoV transmission and N95 respirator use. J Hosp Infect. 2020;105(1):104-5.

39 Sultan S, Lim J, Altayar O, Davitkov P, Feuerstein J, Siddique S, et al. AGA institute rapid recommendations for gastrointestinal procedures during the COVID-19 pandemic. Gastroenterology. 2020;159(2):739-58.e4.

40 Aguila EJT, Cua IHY, Dumagpi JEL, Francisco CPD, Raymundo NTV, Sy-Janairo MLL, et al. COVID-19 and its effects on the digestive system and endoscopy practice. JGH Open. 2020;4(3):324-31.

41 Irisawa A, Furuta T, Matsumoto T, Kawai T, Inaba T, Kanno A, et al. Gastrointestinal endoscopy in the era of the acute pandemic of coronavirus disease 2019: Recommendations by Japan gastroenterological endoscopy society (issued on April 9th, 2020). Dig Endosc. 2020;32(5):648-650.http://dx.doi.org/10.1111/ den. 13703 .

42 British Society of Gastroenterology guidance on recommencing gastrointestinal endoscopy in the deceleration and early recovery phases of COVID-19 pandemic [Internet]. bsg.org. uk. 2020 [cited 2020 May 15]. Available from: https://www.bsg.org.uk/covid-19-advice/ bsg-guidance-on-recommencing-gi-endoscopy-in-the-deceleration-early-recoveryphases-of-the-covid-19-pandemic/ecoveryphases-of-the-covid-19-pandemic/.

43 Infectious diseases society of America guidelines on the diagnosis of COVID-19 [Internet]. Idsociety.org. $2020 \mathrm{https} / / / \mathrm{www}$. idsociety.org/practice-guideline/covid-19-guideline-diagnostics/.

44 Lim SM, Cha WC, Chae MK, Jo IJ. Contamination during doffing of personal protective equipment by healthcare providers. Clin Exp Emerg Med. 2015;2(3):162-7.

45 Lui RN, Wong SH, Sánchez-Luna SA, Pellino G, Bollipo S, Wong MY, et al. Overview of guidance for endoscopy during the coronavirus disease 2019 pandemic. J Gastroenterol Hepatol. 2020;35(5):749-59.

46 Han J, Wang Y, Zhu L, Cui Y, Li L, Zeng Z, et al. Preventing the spread of COVID-19 in digestive endoscopy during the resuming period: meticulous execution of screening procedures. Gastrointest Endosc. 2020;92(2): $445-7$.
47 Corral J, Hoogenboom S, Kröner P, VazquezRoque M, Picco M, Farraye F, et al. COVID-19 polymerase chain reaction testing before endoscopy: an economic analysis. Gastrointest Endosc. 2020;92(3):524-34.e6.

48 Boettler T, Newsome PN, Mondelli MU, Maticic M, Cordero E, Cornberg M, et al. Care of patients with liver disease during the COVID-19 pandemic: EASL-ESCMID position paper. JHEP Rep. 2020;2(3):100113.

49 Targher G, Mantovani A, Byrne C, Wang X, Yan H, Sun Q, et al. Risk of severe illness from COVID-19 in patients with metabolic dysfunction-associated fatty liver disease and increased fibrosis scores. Gut. 2020;69(8):15457. http://dx.doi.org/gutjnl-2020-321611

50 Zhou Y, Zheng K, Wang X, Yan H, Sun Q, Pan $\mathrm{K}$, et al. Younger patients with MAFLD are at increased risk of severe COVID-19 illness: a multicenter preliminary analysis. J Hepatol. 2020;73(3):719-21.

51 Gao F, Zheng K, Wang X, Yan H, Sun Q, Pan $\mathrm{K}$, et al. Metabolic associated fatty liver disease increases coronavirus disease 2019 disease severity in nondiabetic patients. J Gastroenterol Hepatol. 2020.

52 Clinical best practice advice for hepatology and liver transplant providers during the $\mathrm{CO}$ VID-19 pandemic: AASLD expert panel consensus statement [Internet]. aasld.org. 2020 [cited 2020 May 15]. Available from: https:// www.aasld.org/sites/default/files/2020--05/ A ASLD-COVID 19-ClinicalInsightsMay142020-FINAL-v2.pdf.

53 Yip T, Lui G, Wong V, Chow V, Ho T, Li T, et al. Liver injury is independently associated with adverse clinical outcomes in patients with COVID-19. Gut. 2020. http://dx.doi. org/10.1136/gutjnl-2020-321726.

54 Sarin S, Choudhury A, Lau G, Zheng M, Ji D, Abd-Elsalam S, et al. Pre-existing liver disease is associated with poor outcome in patients with SARS CoV2 infection; The APCOLIS study (APASL COVID-19 liver injury spectrum study). Hepatol Int. 2020:1-11.

55 Wong G, Wong V, Thompson A, Jia J, Hou J, Lesmana $\mathrm{C}$, et al. Management of patients with liver derangement during the $\mathrm{COV}$ ID-19 pandemic: an Asia-Pacific position statement. Lancet Gastroenterol Hepatol. 2020;5(8):776-87.

56 Iavarone M, Sangiovanni A, Carrafiello G, Rossi G, Lampertico P. Management of hepatocellular carcinoma in the time of COVID-19. Ann Oncol. 2020;31(8):1084-5.
Gradual Return of Gastroenterology

Practice during the COVID-19 Pandemic
Dig Dis 2021;39:140-149

DOI: $10.1159 / 000511008$ 